Théologiques

Théologiques

\title{
Ville, pratiques religieuses et mission chrétienne
}

\section{André Charron}

Volume 3, numéro 1, mars 1995

Symbolique urbaine et foi chrétienne

URI : https://id.erudit.org/iderudit/602415ar

DOI : https://doi.org/10.7202/602415ar

Aller au sommaire du numéro

\section{Éditeur(s)}

Faculté de théologie de l'Université de Montréal

\section{ISSN}

1188-7109 (imprimé)

1492-1413 (numérique)

Découvrir la revue

\section{Citer cet article}

Charron, A. (1995). Ville, pratiques religieuses et mission chrétienne.

Théologiques, 3(1), 61-88. https://doi.org/10.7202/602415ar

\section{Résumé de l'article}

La ville d'aujourd'hui est celle d'une société urbanisée, qui est à la conjonction des phénomènes de la modernité et de l'industrialisation et où la mobilité et la diversité valorisent les choix de l'individu. Cette situation transforme le rapport au religieux dans les domaines de la culture, des structures d'appartenance et surtout du projet individuel : on en voit plusieurs illustrations à partir du cas du Québec actuel. L'exercice de la mission du christianisme doit dès lors s'ajuster à ces déplacements et l'Église retrouver la dynamique de cet espace public qu'est l'unité de vie collective de la ville. 


\title{
Ville, pratiques religieuses et mission chrétienne
}

\author{
André CHARRON \\ Faculté de théologie \\ Université de Montréal
}

\section{RÉSUMÉ}

La ville d'aujourd'hui est celle d'une société urbanisée, qui est à la conjonction des phénomènes de la modernité et de l'industrialisation et où la mobilité et la diversité valorisent les choix de l'individu. Cette situation transforme le rapport au religieux dans les domaines de la culture, des structures d'appartenance et surtout du projet individuel: on en voit plusieurs illustrations à partir du cas du Québec actuel. L'exercice de la mission $d u$ christianisme doit dès lors s'ajuster à ces déplacements et l'Église retrouver la dynamique de cet espace public qu'est l'unité de vie collective de la ville.

C'est dans la ville que l'on trouve au mieux le contexte global de la réalité humaine d'aujourd'hui. Il importe de prendre la mesure de ce que cela représente, avec un regard positif et sans nostalgie, et d'en tirer quelques conséquences pour une présence chrétienne qui s'insère dans les conditions de la vie urbaine. Devant l'ampleur d'un tel sujet, le présent propos ne peut avoir de prétention à fournir plus qu'une première esquisse. Je choisis de situer d'abord ce phénomène socioculturel qu'est la ville. J'exposerai ensuite les principales transformations du rapport au religieux dans la société urbaine à partir du cas du Québec actuel. Compte tenu de la signification de la ville et des nouvelles pratiques religieuses identifiées, je donnerai un aperçu de l'aménagement souhaitable de la mission chrétienne en tel contexte. 


\section{La ville comme fait de culture}

La ville a tenu un rôle de premier ordre dans l'évolution des peuples. Avec la concentration de la population, des échanges et des services, elle constitue un espace public qui en fait une médiation cardinale de la culture. C'est dans la cité ou la ville que se développent la politique, le commerce, le culte, les arts et les sciences, la mise en ordre de la vie sociale jusqu'à la codification du droit. La ville est le centre du savoir et du pouvoir, exerçant souvent son influence à l'extérieur sur toute une région. En elle apparaissent les grands équipements collectifs tels le marché, le temple, le stade, le théâtre, l'agora, l'hôpital, l'école, la bibliothèque et les archives. La créativité et l'avancement de la culture sont associés à la ville au point que le langage puise aux champs sémantiques de la polis et de la civitas le terme de "politique " pour dire ce qui est relatif aux affaires publiques d'une société organisée, celui de "civilité " pour référer au savoir-vivre régissant la sociabilité, celui de "civiliser" pour signifier l'entreprise de faire passer une collectivité à un état de plus haut développement et, enfin, celui de "civilisation" pour nommer le processus historique de progrès matériel, social et culturel ou pour désigner son résultat en un état social considéré comme avancé ${ }^{1}$. La ville se veut un centre civilisateur visant un idéal d'humanité. Ainsi le pensaient déjà Aristote et Cicéron. La réalité historique est certes plus problématique et prête à des points de vue critiques où s'ajoutent aux lectures optimistes les lectures pessimistes sur la dégradation, l'appauvrissement ou l'inhumanité des villes. Robert Park a une position plus équilibrée : la ville, produit de la nature humaine, est un milieu qui donne à l'individu les plus grandes possibilités de s'affirmer en bien comme en $\mathrm{mal}^{2}$. La représentation de la ville est vue, en somme, comme une synthèse de la civilisation.

La ville est aussi le reflet des mutations de la culture. Le village d'antan était un milieu où chacun connaissait les autres et où les relations interpersonnelles étaient la base de la vie sociale. Cette connaissance mutuelle dans une géographie étroite favorisait un fort contrôle social entretenu par l'observation directe et la solidarité inconditionnelle. C'érait une communauté de type primaire, fondée sur les interactions et l'identifi-

1 Cf. Dictionnaire historique de la langue française, Le Robert. Paris, 1993, articles «Civil », « Politique », "Ville».

2 "The city in short shows the good and evil in human nature in excess", citation de Robert PARK, grand sociologue des villes de l'École de Chicago, dans H. CARRIER, Lexique de la culture pour l'analyse culturelle et l'inculturation. Tournai-Louvain-laNeuve, Desclé, 1992, p. 388. 
cation affective, à l'intérieur de laquelle s'accomplissait l'ensemble des fonctions sociales. En contexte chrétien, la paroisse constituait l'encadrement religieux d'une telle communauté humaine préexistante. Le village était donc un milieu totalisant où l'identité collective l'emportait sur l'affirmation de l'individu. La ville traditionnelle, préindustrielle, offre bien quelques similitudes avec le village au plan de la vie quotidienne, notamment dans les quartiers où souvent le lieu de résidence est aussi celui du travail et où les rapports peuvent demeurer de type communautaire à travers le voisinage et la paroisse. Mais les gens s'y mélangent audelà des quartiers, qui ne sont pas autosuffisants. Avec une mobilité encore restreinte, ils se déplacent d'un quartier à l'autre et ils fréquentent le centre qui anime la vie collective. Le citadin et même l'étranger s'y sentent à l'aise, n'étant plus soumis à un contrôle contraignant. "La personne relève d'influences diverses qui ne se compénètrent plus. Bref, elle cesse d'être encadrée par un groupe totalisant et acquiert une autonomie nouvelle $^{3}$. "Plus encore, la ville comprend une diversité de champs d'activité où la production et l'offre de biens et services matériels, culturels et administratifs passent souvent par des lieux et acteurs spécialisés. Les milieux de vie sont pluriels, les activités diverses et les rôles sociaux variés. La ville structure, coordonne et organise les champs d'activité, y compris ceux qui se trouvent à la campagne de ses environs. Dans un ouvrage récent, J. Remy et $\mathrm{L}$. Voyé insistent ainsi sur la signification fonctionnelle de la ville "comme étant un espace centripète qui s'organise en vue de la structuration et de la mise en interrelation des divers champs d'activité et qui tend à la systématisation et à la formalisation », le centreville jouant un rôle déterminant pour l'organicité de l'ensemble 4 .

D'autres changements vont s'imposer. La ville d'aujourd'hui est celle de la société urbanisée : elle est le produit de l'urbanisation qui est à la conjonction des phénomènes de la modernité et de l'industrialisation. La modernité comme type de civilisation, univers de valeurs et mode de vie, est le facteur majeur des mutations de la culture occidentale des derniers siècles. Elle se comprend dans l'horizon de la transformation du rapport de l'homme à la nature. L'homme fut longtemps dans un rapport de soumission à la nature qui, conçue comme un tout englobant régi par des lois immuables, représentait l'ordre cosmique exemplaire à imiter et à repro-

3 Jean REMY, dans F. HOUTARD et J. REMY, Milieu urbain et communauté chrétienne. Paris, Mame, 1968, p. 152.

4 Jean REMY et Liliane VOYÉ, La ville: vers une nouvelle définition? Paris, L'Harmattan, 1992, p. 39. 
duire. Perçue comme donnée brute et inaliénable, la nature était la matrice de l'ordre social, déterminant la prépondérance du grand tout sur la partie qu'est la personne, du système sur l'individu, de l'ordre sur la liberté, des gouvernants sur les gouvernés. À la faveur des sciences physiques et mathématiques, des découvertes expérimentales et de l'évolution de la technique, l'homme moderne est entré dans un rapport de domination sur la nature, de conquête, d'initiative capable d'innovation et d'invention. Il éprouve ce qu'il a de science et de puissance et se pense une liberté sans commune mesure avec la nature. Cette maîtrise s'étend à la conception de l'homme lui-même. La modernité promeut l'affirmation du sujet humain, l'émergence de sa conscience personnelle, la considération de son historicité, c'est-à-dire de son cheminement en constant devenir. Elle privilégie l'autonomie de la pensée et de la conduite, l'aptitude à faire des choix, l'action transformatrice, en contrepartie des modèles de convention, de soumission et d'imitation de jadis. Elle prône le primat de l'individu sur l'institution, la participation, la concertation et le débat. Or la ville, carrefour de ressources démultipliées, avec ses brassages d'idées, ses innovations et expérimentations de tous genres, est le lieu par excellence où la modernité s'élabore, éclôt, se manifeste.

Facteur de modernisation, le développement des sciences et des techniques se traduit aussi en invention d'outils qui amènent l'industrialisation par l'installation des manufactures, des usines industrielles puis des entreprises de haute technologie. Cela provoque un mouvement de la maind'œuvre vers les villes, qui s'étendent considérablement. L'urbanisation devient intensive et extensive. Mais surtout, l'industrialisation modifie dans la ville les rôles, l'espace et les relations. Par la différenciation de la production, elle implique nécessairement la spécialisation du travail et des tâches. Pour œuvrer, on doit se déplacer en divers endroits du centre ou de la périphérie, d'un quartier à l'autre, de sorte que le lieu du travail est dorénavant séparé du lieu de résidence. Cette spécialisation s'accroît avec la montée du secteur tertiaire qui connaît un essor du côté des services professionnels et même des services à la production, dans les capitales et métropoles comme Montréal ${ }^{5}$. La rationalisation moderne, la poursuite systématique d'objectifs à buts déterminés, atteint l'ensemble des fonctions fondamentales de la vie sociale - famille, résidence, économie, politique, éducation, loisirs, religion - qui deviennent de plus en plus

5 Mario POLEZE, «La transformation des économies urbaines: tertiarisation, délocalisation et croissance économique », CahRechSoc 6/2 (1988) p. 13.25. 75\% des Montréalais travaillent dans le secteur tertiaire et l'activité manufacturière se déplace vers la périphérie. 
spécialisées, répondant chacune à une logique et des intérêts propres ${ }^{6}$. La spécialisation des fonctions engendre la multiplication des institutions et des groupes qui ont leur localisation en des endroits différents et qui renvoient à une variété de rôles sociaux. «Le contrôle social devient de ce fait partagé et diffus, chaque groupe n'exerçant sur l'individu qu'un contrôle limité au rôle qui lui est assigné. Cette situation provoque chez l'homme des villes une réaction de refus vis-à-vis de toute institution qui désirerait contrôler l'ensemble de sa vie ${ }^{7}$. » Et encore l'urbain lui-même est amené à remplir ses divers rôles selon des appartenances différentes.

La mobilité est une caractéristique première de la société urbanisée. J. Remy et L. Voyé définissent l'urbanisation comme "processus intégrant la mobilité spatiale à la vie quotidienne à partir et des possibilités techniques de se déplacer et d'une lecture positive de la mobilité ${ }^{8}$ ". Elle structure la vie des individus er des acteurs collectifs qui se constituent à travers des espaces multiples. Les équipements collectifs sont dispersés dans un espace discontinu et une pléiade d'activités sont offertes. Les relations se font en divers endroits. La mobilité devient donc une condition d'adaptation et de participation à la vie urbaine. Les auteurs avancent que la principale logique d'appropriation du citadin urbanisé est la valorisation du choix de l'individu qui a la liberté de se comporter à sa guise. Au plan de la personnalité, c'est le projet individuel qui prévaut, au cœur de la pluralité des milieux et des sollicitations. Au plan social, c'est l'individu qui est au centre du rapport d'échanges et son projet personnel se substitue au projet collectif ${ }^{9}$. En plusieurs domaines, les relations sont fonctionnelles, c'est-à-dire qu'elles s'établissent en regard de la compétence affichée, de l'objectif visé, de l'utilité qu'on en tire, sans implication affective. Ce type de relations n'est pas pour autant sans signification humaine, car la qualité du service est mieux garantie, le poids d'obligations ou de pressions qu'entraînent souvent les liens de personne à personne se trouve écarté et l'autonomie du choix est davantage préservée. Cependant, les relations interpersonnelles ne sont plus l'élément-clé de l'intégration sociale comme autrefois. Les relations sont plutôt disloquées selon les différentes fonctions auxquelles on s'adonne; elles sont par-

6 Même la résidence n'y échappe pas, que l'on choisit (du moins chez les classes autres que populaires) selon l'évolution de la famille, de ses besoins, de ce qui satisfait ses membres.

7 François HoUTART, dans F. HoutART et J. REMY, op. cit., p. 59.

8 J. REMY et L. VOYÉ, op. cit., p. 11, 59, 63, 76.

9 Ibid., p. $80,87$. 
tielles. Mais on voit aussi se multiplier des petits groupes d'intérêt commun, d'entraide gratuite, de soutien réciproque, "auxquels on a délibérément choisi de participer et dont on sait que l'on peut sortir à tout moment sans pour autant désorganiser l'ensemble de son réseau interactionnel ${ }^{10}$ ". L'individu pouvant participer à plusieurs groupes tient un certain nombre de solidarités partielles, chacune renvoyant à une activité particulière. Il reste que, face à l'anonymat et au nouveau nomadisme prédominant dans l'univers urbain, ces noyaux de relations groupales contribuent à l'intégration du projet individuel.

Les effets de l'urbanisation rejoignent aussi les campagnards. Le village d'aujourd'hui participe à "un espace régional de mobilité ". Il est concerné par la transformation du régime d'échanges de tous ordres et il a besoin de la ville et des autres villages pour le partage des ressources. Mais surtout la mentalité est influencée par le principe de l'autonomie de l'individu. Car la culture urbaine est diffusée partout par les médias de communication qui rayonnent précisément à partir de la ville.

L'urbanisation et ses composantes de modernité et d'industrialisation provoquent ainsi l'affaiblissement des communautés d'appartenance. On parle même de l'éclatement des communautés naturelles, jusqu'à celle de la famille qui subit des pressions concurrentielles en termes d'exigences de rôles à tenir, de valeurs et d'investissement affectif. On y partageait une vision commune du monde, même dans la paroisse traditionnelle qui coincidait avec la communauté villageoise.

Or dans une société urbanisée, dès lors que la ville suppose l'éclatement de ces solidarités primordiales pour l'apprentissage de relations fonctionnelles élargies, $[\ldots]$ les communautés sont remplacées par des réseaux multiples d'influence, de clientèle, d'amitié, de soutien, voire de partage. Ces réseaux, générateurs de solidarités partielles et éphémères, constituent une autre façon de faire société, non plus en diffusant des valeurs et des modèles de haut en bas mais en les explicitant par ramification de proche en proche, sur le terrain. On ne peut donc parler désormais que de communautés construites, sélectives ${ }^{11}$.

10 Ibid. p. 88.

11 Raymond LEMIEUX et Jean-Paul MONTMINY, «La vitalité paradoxale du catholicisme québécois", dans G. DAIGLE (dir.), Le Québec en jeu. Comprendre les grands défis, Montréal, Presses de l'Université de Montréal, 1992, p. 589-590. 
La «ville urbanisée " offre d'énormes possibilités: ressources riches en personnes, en éducation et en biens, variété, liberté, tolérance, complémentarité des tâches dans l'interdépendance, circulation des informations, axes de communication sélectifs facilitant une nouvelle sociabilité et même une nouvelle solidarité communautaire. Toutefois, selon le modèle capitaliste dominant, l'accent mis sur le projet individuel suppose des stratégies personnelles dont la médiation nécessaire est l'argent. Les jeux du libéralisme économique s'appuient sur cette logique. La mobilité et l'individualisation ne profitent donc pas à tous de la même façon. L'exclusion d'un grand nombre dans l'accès aux ressources urbaines, conséquence du chômage ou de l'emploi précaire à temps partiel, crée des inégalités et la disparité des classes sociales dont le résultat est une société "cassée en deux». En milieux appauvris, "dans un univers aux horizons incertains, certains efforts pour s'en sortir peuvent paradoxalement déboucher sur la criminalité, les toxicomanies, la violence, ou des comportements qui contribuent à marginaliser davantage ${ }^{12} \%$. La ville est aussi un lieu de contraintes et d'aliénations. Mais des solidarités communautaires naissent pour répondre aux urgences, le plus souvent dans le voisinage du quartier. Car, alors que les gens instruits et à l'aise ont un réseau plutôt déterritorialisé, pouvant s'offrir tous les échanges et services là où ils ont l'opportunité d'aller les chercher, les gens de classe populaire et moyenne ont un réseau territorialisé, proche ${ }^{13}$. Cette émergence des réseaux de voisinage n'est pas, par ailleurs, limitée aux défavorisés. Une recherche d'Andrée Fortin montre que des réseaux d'entraide et de convivialité, selon les filières de la famille et des amis, existent en banlieue, dans les quartiers populaires et chez les gens qui font la reconquête du centre-ville. Ces réseaux de sociabilité sont liés à l'espace, par bouts de rue ou coopératives d'habitation. Si l'on tend à se rapprocher des gens avec qui on a des affinités, on y vit toutefois en îlots isolés et on n'a pas idée de ce qui se passe de l'autre côté de sa rue. Les réseaux ont tendance à s'ignorer mutuellement. "La ville est le lieu des réseaux, mais n'en est pas un elle-même; elle n'est pas un réseau de réseaux, mais leur juxtaposition 14 . "

12 Suzanne LA FERRIĖRE, “ La dynamique communautaire montréalaise ", pièce au dossier de la série « Montréal cassé en deux ", Relations 584 (1992) p. 240.

13 Andrée FORTIN, “Du voisinage à la communauté , dans un dossier consacré à la reconquête de la ville, CahRechSoc 6/2 (1988) p. 159. Cette recherche est faite à partir de 12 quartiers de Québec et refère à d'autres études portant sur le milieu montréalais.

Ibid. , p. 159. 


\section{Les mutations du rapport au religieux dans la ville urbanisée}

La «ville urbanisée", comme fait de culture, provoque des transformations dans les rapports au religieux. Cela se laisse voir d'abord au plan des structures. La paroisse territoriale fut longtemps l'institution normalisée de la pratique religieuse et de la vie chrétienne communautaire. Elle est l'héritière en Amérique de l'organisation ecclésiale européenne depuis le haut Moyen Âge où elle est née des conditions d'aménagement de l'espace rural. Alors que, aux premiers siècles du christianisme, c'était l'Église épiscopale d'une ville tout entière et indivisible qui était appelée "paroisse", évoquant sa demeure en ce lieu d'habitations global, c'est lorsque les activités se développèrent dans les campagnes et seigneuries qu'on institua de petites paroisses différenciées sur autant de territoires restreints. Suivant un quadrillage systématique des campagnes, on établit des paroisses autosuffisantes et distincres de la ville épiscopale pour encadrer la fidélité religieuse de chaque communauté de bourgs puis de villages. Plus tard, on transplanta ce modèle rural en ville, en y reproduisant la division en paroisses territoriales selon le même principe du quadrillage qu'on adopta pour les quartiers ${ }^{15}$.

En Nouvelle-France, Montréal, constitué en paroisse en 1678, s'étendit en absorbant les faubourgs et les "côtes " agricoles (unités de base de la campagne montréalaise) qui furent des dessertes de l'unique paroisse Notre-Dame jusqu'au milieu du $\mathrm{XIX}^{\mathrm{e}}$ siècle pour être ensuite reconnues en paroisses autonomes sous Mgr I. Bourget. À la fin du XIX siècle, Montréal, Québec et quelques autres villes grossissant avec la première vague d'industrialisation, l'Eglise y ramifia son encadrement en découpant "les quartiers urbains en paroisses dans l'espoir que celles-ci continueront d'être les cellules de base de la collectivité québécoise et les racines de l'église-institution ${ }^{16}$ ". Cette pratique va continuer jusque dans les années 1950-1960, notamment à Montréal où le cardinal P.-É. Léger crée de nombreuses paroisses dans l'intention de rapprocher le pasteur et les fidèles. On a donc en ville des paroisses héritées du modèle rural qui représentent autant de territoires découpés dans le tissu urbain. Or depuis le début du XXe siècle, elles n'encadrent pourtant plus des milieux

15 André ChARron, «L'Église : de la maison à la ville », dans J.-C. PETIT (dir.), "Où demeures-tu? *. La maison depuis le monde biblique. Montréal, Fides, 1994, p. 444 ss.

16 Nive VoISINE, Histoire de l'Église catholique au Québec 1608-1970, (Commission d'étude sur les laïcs et l'Église), Montréal, Fides, 1971, p. 65. 
homogènes et autosuffisants. "L'émergence de milieux de travail, de milieux scolaires, de milieux de loisir en dehors de leurs frontières les ouvrent au vent du large, substituant au sens paroissial un sens d'appartenance à la cité ou à la région ${ }^{17}$. " Aujourd'hui, en effet, les paroisses ne peuvent plus résister au facteur de globalisation des relations dans l'ensemble urbain, qui ne se cantonnent plus à l'intérieur de leur territoire restreint. Même au plan religieux, la mobilité du citadin fait qu'il va chercher en divers endroits de la ville ses services là où ils sont pour lui les plus significatifs, compétents ou commodes. Les pratiques paroissiales ne pourront rester autarciques quand la population les considèrent relativisées.

La structure paroissiale subit d'ailleurs le contrecoup de la différenciation du monde urbain en de multiples univers sociaux pour les activités desquels le citadin est sollicité de toutes parts. Cela occasionne même l'éclatement des appartenances, qui se trouvent ébranlées, affaiblies ou à tout le moins partagées entre plusieurs pôles. La paroisse urbaine ne repose plus sur un substrat communautaire unifié. Elle représente un univers parmi d'autres et ses activités sont soumises au jeu de la concurrence. En outre, dans des quartiers de centre-ville, les déménagements fréquents, la forte proportion de chambreurs ou de locataires d'étroits appartements à grande mobilité par rapport au nombre de familles stables et, plus encore, la désintégration sociale qu'entraînent le chômage, l'exclusion, l'indigence, les ruptures familiales, risquent de rendre indifférent à la vie collective et dès lors à la vie paroissiale : c'est dans ces paroisses de centre-ville que s'observent les plus faibles taux de pratique dominicale, extrêmement bas ${ }^{18}$. Pour l'un ou l'autre de ces facteurs, la paroisse territoriale urbaine éprouve la désertion, sinon l'indifférence. $E_{t}$ les pratiques religieuses institutionnalisées, normalement reliées à l'intégration paroissiale, sont raréfiées.

La société urbanisée s'est sécularisée. Son virage moderne impliquait la sécularisation, ce processus d'émancipation culturelle de la tutelle des Églises et du contrôle religieux. La sécularisation va opérer rapidement au cœur de la Révolution tranquille selon deux axes principaux : la fin du monopole et du contrôle des institutions d'Église sur la vie sociale et la récession du poids prédominant des visions ou significations religieuses

17 Jean HAMELIN, Le XXe siècle, tome 2: De 1940 à nos jours, (Histoire du catholicisme québécois). Montréal, Boréal express, 1984, p. 53.

18 Voir R. LEMIEUX, «La pratique religieuse ou les défis d'une Église urbaine», dans Jean-Paul II, une Église au rendez-vous. Montréal, Paulines, 1984, p. 58. 
sur la vie collective. C'est à partir de Montréal et de Québec, foyers principaux de la culture québécoise, que le mouvement fait son chemin. Les intellectuels, leaders d'opinion et responsables sociaux - avec l'accord tacite ou la collaboration des clercs ouverts aux perspectives du concile Vatican II - imposent l'autonomie des structures objectives de la société, chacune obéissant aux lois propres de son domaine: organisation politique, néo-nationalisme, droits humains fondamentaux, philosophie, éthique, production culturelle et scientifique, éducation; déconfessionnalisation des syndicats, hôpitaux, services sociaux, associations, collèges et universités, commissions scolaires. Ce type de sécularisation n'a pas pour autant aboli la religion. Certes, des recherches et publications sont inféodées au rationalisme techno-scientifique, ignorant ou refoulant les dimensions spirituelles et religieuses de la personne. Certes, des rapports humains sont sacrifiés à la raison instrumentale axée sur la performance technique, l'efficacité et la productivité, sans considération des fins transcendantes du projet humain. Mais, même si le champ religieux est porté à la marge de la vie publique, la religion n'a pas disparu de la culture profonde entendue comme complexe de références pour la conduite de la vie, pour la réflexion et l'action. Cependant l'expression religieuse, ne faisant plus partie de la nécessité sociale, est renvoyée dans la sphère de la vie privée. On le constate jusque dans les grands médias urbains qui accordent de moins en moins de place au fait religieux. Plusieurs individus hésitent à s'afficher chrétiens dans leurs relations publiques. La pratique religieuse se fait au titre de la vie privée. Et la plupart des croyants demeurent très jaloux dans leur vie même des catégories et des valeurs de la sécularité qu'ils veulent reconnues telles.

La métropole du Québec tient le rôle pivot dans ce qu'on a appelé la reconquête francophone portée par la Révolution tranquille depuis les années $1960^{19}$. On s'ouvre aux courants modernes étrangers, on revendique la liberté de pensée, on désire se donner ses propres leviers et ses propres règles. Pour l'affirmation neuve des individus et du pays, comme dit le poète $P$. Chamberland, on a à exorciser "l'ange anglais et romain ", à ranimer "cette petite étoile étouffée dans l'écrin d'encens 20 \%. Le rattrapage culturel paraît, en effet, inséparable d'une volonté de libération d'un passé religieux aliénant. La critique de la religion s'affirme dans la poésie, le roman, le théâtre, les essais, la chanson. Elle dénonce la 
convention de l'orthodoxie laissant peu de place à l'interrogation et à la recherche, la spiritualité rétrécie du renoncement aux plaisirs mondains, la morale de l'interdit, le spectre de la culpabilité, le formalisme de positions ecclésiastiques trop peu attentives aux drames humains et aux sensibilités séculières, le désuétude du langage religieux par rapport à une culture qui fait la conquête de sa modernité. Cette pratique critique est partie prenante de l'entreprise de désencombrer l'héritage, de réouvrir les questions, de renouer avec soi, avec ses manques et ses doutes, de réapprendre à marcher, pour le projet d'un homme nouveau en mal de s'épanouir, de goûter la vie, de prendre en charge son humanité, dans l'espace libre du croire. Telle est la thématique d'un courant de fond de la production culturelle où derrière les rejets s'affirment des requêtes. Elle répercute le sentiment de bien des gens mais en même temps le façonne.

La modernité urbaine n'est pas sans amener des transformations chez les personnes elles-mêmes. Elle renforce l'individualisme. Non pas tellement parce que la ville amenuise le soutien communautaire et isole l'individu. Mais parce que la mobilité et la diversité urbaines obligent l'individu à faire des choix personnels et le confrontent à sa liberté. Et plus profondément parce que l'affirmation du sujet humain et l'autonomie de sa pensée et de sa conduite sont bien les assises anthropologiques de la modernité. La ville n'enferme ni ne contrôle; elle ouvre aux influences disparates, favorise l'émancipation du sujet autonome et l'individualisation du projet de vie. L'individualisme est cette tendance valorisant les droits et responsabilités de l'individu, la réflexion personnelle, la possibilité de faire des choix et de maîtriser son existence. Il comporte, certes, les risques ou les dérives de la subjectivité en narcissisme, en repliement sur soi, rupture d'avec l'altérité ou anomie, voire en égoïsme. Mais, ainsi que l'avance C. Taylor, dans une éthique de l'authenticité, l'individualisme se comprend comme l'idéal moral de l'accomplissement de soi, renvoyant l'individu à sa source intime, à la recherche de son identité originale ${ }^{21}$. En matière religieuse, le sujet urbain est ainsi de plus en plus renvoyé à ses convictions personnelles. Cela se comprend d'autant que l'on tente de passer au Québec d'une société de la prescription où c'était les institutions qui prescrivaient aux individus les devoirs, consignes, sens et pratiques, à une société de l'inscription où les individus devenus sujets doivent se choisir et se déterminer eux-mêmes une échelle de valeurs et un mode d'inser-

21 Charles TAYLOR, Grandeur et misère de la modernité. Montréal, Bellarmin, 1992. 
tion dans le monde. L'individu-sujet cherche à ne plus être défini par procuration via les institutions mais, face à lui-même, "il est à une croisée des chemins difficile où les indications pour opérer un choix se font très rares 22 ". Ce profond changement se réalise dans tous les domaines de la vie en société, y compris dans ceux de la vie religieuse et ecclésiale. La logique de l'inscription est incontournable et la quête de sens est relancée.

Le milieu urbain est aussi hétérogène et pluraliste. Le Montréal métropolitain comprend une pluralité de confessions : $77,5 \%$ de catholiques, $8,2 \%$ de protestants, $2,7 \%$ d'orthodoxes, $3,1 \%$ de juifs, $1,3 \%$ de musulmans, $0,9 \%$ de bouddhistes, $0,4 \%$ d'hindous 23 . On y voit en outre un foisonnement de "nouveaux mouvements religieux " constitués en dehors des grandes religions historiques, qui intéressent $3 \%$ de la population mais dont les adeptes ne dépassent pas $1 \%$. R. Bergeron les classe en trois familles : les mouvements de restauration du christianisme de type fondamentaliste voulant le refonder sur une conception autoritariste de la Bible sans égard à la rationalité moderne, puis ceux de type intégriste opposés au devenir historique et refusant la modernité séculière; les mouvements de rénovation spirituelle fusionnant des éléments empruntés aux religions orientales, à l'ésotéro-occultisme, à la psychologie humaniste, à la science-fiction pour proposer l'odyssée gnostique solitaire " où chacun prend le chemin du moi pour aboutir au Soi et à la fusion divine "; le mouvement du Nouvel Âge, structuré autour d'une métaphysique moniste, d'une anthropologie gnostique et d'une épistémologie d'inspiration ésotérique, appuyant une spiritualité cosmocentrique et une religiosité magique de réenchantement du monde ${ }^{24}$. Enfin 5,4\% des Montréalais se déclarent "sans religion 25 ": cette catégorie indifférenciée comprend des agnostiques, des athées et des indifférents à toute religion. Il y a en effet des «incroyants» dont l'option fondamentale se précise dans le rejet ou en l'absence de la foi religieuse : ils ont généralement une foi de type

22 Céline ST-PIERRE, «Après la crise de l'engagement, la responsabilisation sociale ", Relations (1987) p. 311-313.

23 Calcul fait à partir des données du recensement de 1991, parues dans Religions au Canada. Statistique Canada, 1993, p. 62-64.

24 Richard BERGERON, «Les sectes religieuses à Montréal », dans G. LAPOINTE (dir.), Société, culture et religion à Montréal : XIXe-XXe siècles. Montréal, VLB éditeur, 1994, p. 186-203.

25 Selon Statistique Canada, op. cit., qui en compte 3,4\% dans le Québec-métro et 3,8\% au Québec. 
humaniste 26 . On retrouve donc une pluralité de positions en matière religieuse, sans oublier, à l'intérieur du catholicisme, la variété des modèles selon les familles d'esprit et les différences de degré d'adhésion. L'acceptation sociale de cette pluralité consacre le pluralisme religieux comme coexistence d'une multiplicité de significations globales et comme état d'esprit qui en résulte. Il normalise la diversité de fait, positivement valorisée bien que vécue en tension. Il implique la diversité des représentations objectivées de l'expérience transcendantale commune et dès lors leur relativisation. Il évite par ailleurs le nivellement informe car il accorde une place à chaque tradition ou orientation.

La pluralité religieuse est accentuée dans la métropole du fait du grand nombre de communautés ethnoculturelles qui correspond au quart de sa population. Bien que plusieurs groupes ethniques soient de foi chrétienne, cela est un facteur déterminant de la pluralité des croyances et des traditions. La ville se veut une société ouverte et pluraliste, même si le christianisme, tradition de la majorité de ses citoyens, est la référence religieuse principale inscrite dans ses racines historiques, ses monuments architecturaux, des œuvres artistiques et littéraires, des valeurs et symboles qui ont façonné un mode de vie, la manière de penser le rapport à la transcendance. De leur côté les citoyens, y compris ceux qui de culture non occidentale sont habitués à ce que culture et religion soient en symbiose, ont à s'intégrer à la culture publique pluraliste. Ils doivent y accepter la manière qu'a cette culture d'accueil de comprendre l'orientation religieuse, à savoir la distinction entre la culture publique commune et la religion, où la confession religieuse est une option personnelle dégagée de l'appartenance ethnique - ce qui ne leur enlève ni le droit ni le fait de vivre selon leur conception ethnoreligieuse au sein de leur communauté culturelle particulière. Mais cette distinction entre culture civique et confession religieuse est à la base des accommodements à consentir, et en certains cas de réglementations légitimes à faire, en matière de tenue vestimentaire, de prescriptions alimentaires, de congés liés aux fêtes religieuses et en regard des normes de santé, de la codification du travail et des conditions d'efficacité du fonctionnement des organisations, pour un vivre ensemble dans l'équilibre du bien commun, ID., "L'indifférence religieuse ", dans R. LATOURELlE (dir.), Dictionnaire de théologie fondamentale. Montréal, Bellarmin; Paris, Cerf, 1992, p. 629-640. L'enquête Bibby décompte $20 \%$ de "sans religion " au Québec mais spécifie que $10 \%$ d'entre eux reviennent à leur religion d'affiliation. 
l'harmonie et la paix sociales. Somme toute, le pluralisme, autant que l'individualisme, renforce la logique de l'inscription.

Voyons de plus près les comportements des individus à l'intérieur même du catholicisme. L'affiliation à la religion d'origine demeure stable. Ainsi $91 \%$ des Québécois catholiques disent maintenir leur rattachement au catholicisme. Mais le membership ecclésial est faible : $25 \%$ se reconnaissent un statut de membres effectifs des paroisses locales 27 . On constate ici encore un notable déplacement : la prise de distance par rapport aux lieux institués d'Église. Plusieurs sont devenus abstentionnistes, sous forme de retrait partiel ou total ${ }^{28}$ : l'Église fait difficulté par son discours culturellement décalé, ses lois disciplinaires intransigeantes ou ses pratiques banales. Certains ont un contentieux à liquider relatif au modèle culturel de religion qui leur fut jadis imposé. Plusieurs se méfient d'une institution qui encadrerait leur vie et se veulent des " chrétiens autonomes ». D'autres sont devenus indifférents aux activités de leur religion d'affiliation.

Le taux de pratique dominicale, pour l'ensemble des catholiques québécois, s'établit entre $30 \%$ et $35 \%$ selon les sondages. Une enquête récente de la firme SOM auprès des catholiques francophones du Montréal métropolitain indique les taux suivants : $23,2 \%$ ont une pratique régulière ( 40 fois et plus par année); $14 \%$ une pratique modérée (de 10 à 39 fois ); $29,5 \%$ une pratique irrégulière ( 1 à 9 fois ); 33,3\% n'ont jamais cette pratique $^{29}$. Chez les pratiquants (au moins une fois l'an), on retient quelques points significatifs : $54 \%$ seulement sont d'accord avec l'idée d'aller à la messe le dimanche; $63 \%$ ne jugent pas important l'accueil à l'église lors des célébrations; $61 \%$ n'estiment pas important d'y rencontrer des connaissances; $80 \%$ privilégient le recueillement à la messe; $59 \%$ sont d'accord qu'on se confesse une fois l'an; $78 \%$ ne sont pas d'accord pour que l'on refuse de baptiser les enfants des non-pratiquants, $75 \%$ que l'on refuse de marier les non-pratiquants, $81 \%$ que l'on refuse des funérailles religieuses à des non-pratiquants. On remarque là des écarts par rapport soit aux coutumes de l'Église-institution, soit aux pratiques

27 Reginald W. BIBBY, La religion à la carte. Pauvreté et potentiel de la religion au Canada. Montréal, Fides, 1988. et «La religion à la carte au Québec : une analyse de tendances ", Sociologie et sociétés XXII/2 (1990) p. 133-144.

28 Cf. Paul-André TuRCOTTE, Intransigeance ou compromis. Sociologie et histoire du catholicisme actuel (Héritage et projet 51). Montréal, Fides, 1994, p. $37-58$.

29 Pierre BOUCHARD, « La pratique religieuse chez les catholiques francophones de Montréal », Prêtre et pasteur 94/1 (1991) p. 38-45. 
communautaires, soit aux consignes de maints agents pastoraux. Par ailleurs, ils sont à l'aise pour communier à $90 \%$, sont d'accord avec l'idée de faire baptiser les enfants à $93 \%$, de se marier à l'église à $84 \%$ et d'avoir des funérailles religieuses à $86 \% 30$. Dans la pratique de ces urbains, il y a donc un déplacement de la cohérence institutionnelle prescrite à une apparente cohérence personnelle vécue.

Selon l'enquête Bibby, les croyances fondamentales perdurent : $90 \%$ des Québécois disent croire en Dieu, $82 \%$ en la divinité de Jésus, $64 \%$ en la vie future; si l'on considère les seuls catholiques, $96 \%$ disent croire en Dieu, $90 \%$ en la divinité de Jésus, $69 \%$ en la vie après la mort. Cependant, pour l'ensemble des Canadiens incluant les Québécois, le résultat cumulatif des croyances, pratiques, expériences et savoirs indique que la configuration religieuse n'est pas intégrée. En effet, si l'on rajoute aux trois grandes croyances la pratique de la prière en privé, l'expérience de la présence de Dieu et les connaissances religieuses élémentaires, seulement $20 \%$ des gens adhèrent à l'ensemble. Par ailleurs, plus de $90 \%$ des adultes québécois demandent les rites liturgiques de passage (baptême, mariage, funérailles) et les jeunes anticipent y avoir recours dans la même proportion. Le sociologue y voit une religion fragmentée de croyances isolées et de pratiques occasionnelles. Il nomme "religion à la carte " cette mosaïque fragmentée où l'on sélectionne, comme sur un menu à la carte, ce qu'on retient et ce qu'on laisse de côté. À ces fragments peuvent se mêler d'autres fragments venant des gnoses, sectes et mouvement du Nouvel Âge. D'après Bibby, les facteurs socioculturels qui, dans les grandes villes, ont rendu fonctionnels les fragments, se sont infiltrés dans les petites villes et n'ont pas épargné les campagnes ${ }^{31}$.

La recherche réalisée par l'équipe de $\mathrm{R}$. Lemieux dans la région urbaine et périurbaine de Québec constate plus avant l'éclatement des croyances qui meublent l'imaginaire individuel des représentations. Elle a repéré les énoncés de croyance recueillis selon quatre pôles: les croyances religieuses, principalement chrétiennes, en $45 \%$ des énoncés; les croyances de type cosmique, tirées des cosmogonies contemporaines et traduisant une vision immanente et totalisante de l'univers, en $25 \%$ des énoncés; les croyances renvoyant au moi intérieur sublimé et capable de tout résoudre, regroupant $18 \%$ des énoncés; les croyances de type social

30 SOM inc., Etude sur la pratique religieuse des catholiques de la région métropolitaine de Montréal pour les Pères du Très Saint-Sacrement. Montréal, mars 1990.

31

R. BIBBY, op. cit., p.104-105, 124, 130. ID., loc. cit. , p. 133-144. 
réifiant des valeurs susceptibles de sauver l'humanité comme la paix, l'amour, la justice, le progrès, pour $11 \%$ des énoncés. Si pour la majorité les croyances demeurent à dominante chrétienne, elles sont souvent assorties de sous-dominantes appartenant aux types du cosmique, du moi ou du social, jusqu'à en être parfois redéfinies ou complètement transformées. La représentation de Dieu elle-même épouse la structure globale de ces croyances éclatées. Les contenus signifiés sous le signifiant "dieu" reçoivent des formulations diverses et la représentation chrétienne de Dieu comme être personnel n'est pas partagée, d'après cette étude, par la majorité des répondants ${ }^{32}$. Cela invite à penser que les transformations subies dans le christianisme vécu sont plus profondes que les seules difficultés touchant les institutions.

Sur l'individualisation de la figure religieuse et sur le comportement sélectif eu égard aux croyances et pratiques, les diagnostics sont variés. On peut y déceler un comportement économique : devant les vitrines de croyances de tous horizons, chacun fait son marché selon ses besoins. Les croyances sont adoptées comme relatives, transitoires, éphémères et elles ne structurent pas toute la vie. Elles ne correspondent pas à une logique objective, rationnelle ou doctrinale, mais elles contribuent à des cohérences subjectives qui se font et se défont selon qu'elles affectent les individus. La cohérence se reconstitue selon les effets que ces croyances produisent: effets de l'utile, de l'expérimenté et du sensé ${ }^{33}$. On associe encore à l'idée de la consommation celle du bricolage, l'individu se fabriquant une religion à sa guise, qui ne dérange pas. On ne saurait oublier pourtant que la sélection est aussi le fait de la plupart des chrétiens qui restent engagés dans leur foi, surtout chez les plus exigeants. La sélection est chez plusieurs un exercice de lucidité et une manifestation de réelle autonomie qui n'ont rien d'une esquive. Il y a la dissidence critique des plus informés, la dissidence savante de maints intellectuels dont celle de théologiens. Il y eut la dissidence du Renouveau charismatique du côté surtout des femmes où, à la faveur du charisme redécouvert comme " maîtresymbole " de la promotion individuelle, des fidèles s'opposaient aux modèles institués de régulation par des stratégies d'autogestion du culte et une socialité d'affinité élective. Malgré quelques dérives subjectivistes et

32 R. LEMIEUX, "Les croyances : nébuleuse ou univers organisé? », dans R. LEMIEUX et M. MILOT (dir.), Les croyances au Québec. Esquisses pour une approche empirique (Les cahiers de recherche en sciences de la religion, 11). Québec, Université Laval, 1992, p. 65-71. Dans le même rapport, R. RiCHARD, "Le Québécois et les métaphores de son Dieu », p. 248.

33 R. LEMIEUX, ibid., p. 71-83. 
quelques emportements alléluiatiques, ce mouvement leur fut une occasion de ressourcement spirituel et de redécouverte de l'Église avant d'être redressé par l'expertise cléricale ${ }^{34}$. Enfin des chrétiens s'imposent une réserve. D'aucuns se demandent si leurs croyances ont de la pertinence pour leur projet de vie et pour leur situation au sein de la culture actuelle. Ils ont de la difficulté à gérer ce rapport. Ils ont perdu un langage convenu et recherchent un nouveau langage. Dans l'univers urbain moderne, ils éprouvent la déconstruction de la cosmologie traditionnelle du christianisme. Ils sont dans un silence fait de prudence et de pudeur. Comme disait $M$. de Certeau, un silence "par nécessité intérieure, pour retourner à un point zéro du langage, pour laisser le désir tâtonner dans les préalables qui rendront possible un réemploi différent, un épurement de nos multiples lexiques. [...] Tous nous attendons des paroles nées de cette nuit, décapées et refaites par ce silence ${ }^{35}$ ".

Les comportements religieux s'exercent souvent hors des lieux de l'Église, de ses offres d'encadrement et de ses normes explicites. Qu'en est-il dès lors de la situation religieuse du grand nombre? Il apparaît qu'on ne puisse diagnostiquer un simple christianisme résiduel. À l'extrême, on ne peut recourir à l'hypothèse d'une "religion invisible " où les individus se créent privément des systèmes de signification de rechange $^{36}$, car ils réfèrent encore pour ce à l'Église et y demandent à tout le moins les rites de passage. On ne peut emprunter le concept de "religion civile", théorie élaborée aux États-Unis d'une religion nationale utilisant dans l'espace public les symboliques judéo-chrétiennes de la transcendance et de la vie après la mort aux fins d'idéaux sacrés représentant la société ${ }^{37}$. Il n'y a pas de chose telle en notre pays qu'une

34 Pauline CÔTÉ et Jacques ZYLBERBERG, « Univers catholique romain, charisme et individualisme: les tribulations du renouveau charismatique canadien francophone ", Sociologie et sociétés XXII/2 (1990) p. 81-94.

35 M. DECerteau et J.-M. DOMENACH, Le christianisme éclaté. Paris, Seuil, 1974, p. $49-50$.

36 R. W. BiBBY, La religion à la carte, p. 64.

37 «Religion élaborée à côté des religions organisées et s'en distinguant nettement » ... où l'ultime souveraineté est attribuée à Dieu, fondant les droits et donnant à la vie politique une fin transcendante, ... la religion civile " est une expression authentique de la réalité religieuse universelle et transcendante, telle qu'elle peut être saisie dans l'expérience du peuple américain ». D'après Robert BELLAH, «La religion civile en Amérique », Archives de Sciences sociales des religions 18/35 (1973) p. 7, 10, 17. Robert TESSIER expose bien les principes de cette théorie dans son volume Déplacements du sacré dans la société moderne. Montréal, Bellarmin, 1994, p. 167-210. 
sacralisation de la nation et les références des individus ne se réduisent pas à ce type de sacré. On n'en est pas non plus à la " religion séculière ", déplaçant le religieux sur les valeurs de l'humanisme séculier ou portant à l'absolu celles de la productivité, de la technique et du progrès du néolibéralisme fonctionnel ${ }^{38}$. Les comportements des individus disent encore leurs références à une culture héritée, même en plusieurs de ses aspects confessionnels.

Les sociologues J.-P. Rouleau, R. Lemieux et J.-P. Montminy diagnostiquent la persistance d'un "catholicisme culturel ", sorte de référent commun inhérent à l'identité culturelle québécoise. Il devient "le référent axial de l'appropriation de la culture " pour l'affirmation de valeurs pérennes spontanément reliées au catholicisme. Il est une culture primordiale où n'est pas exclu le lien à l'Église qui l'a façonnée. C'est une religion diffuse, ni institutionnelle, ni intégrale, évoluant hors du contrôle de l'Église. Le catholicisme culturel répond cependant aux besoins d'appuyer l'existence fragile et "d'inscrire ses valeurs et ses choix dans une tradition, c'est-à-dire dans un ordre symbolique qui a fait ses preuves, même si l'on en critique l'institutionnalisation actuelle, même si l'on en aménage les règles selon ses convenances personnelles 39 ". Je dirai que c'est ainsi un catholicisme de référence plutôt que d'appartenance entretenue. Référence à l'origine commune d'un peuple de baptisés, à l'héritage familial fait de croyances et de valeurs chrétiennes, au patrimoine collectif; elle comprend un répertoire, un lexique, des rites, des symboles et plus profondément des attitudes de foi et de charité. Mais déficit d'appartenance au complexe organique et intégral des croyances et des pratiques officiellement proposées, aux consignes et aux lieux d'Église, aux interactions entre membres des communautés.

Dans l'enquête menée par M. Milot auprès de parents ayant un enfant à l'école primaire, ceux-ci affirment leur identification presque systématique au catholicisme comme à une culture primordiale qui tient de leur héritage familial et de la référence à la communauté catholique. Ils veulent que soit transmise à l'enfant une "base religieuse " capable de

Le recours à ce type de sacré permettrait, dit-on, de dépasser l'individualisme et d'assurer le rapport équilibré entre les idéaux d'autonomie et de solidarité.

38 R. LEMIEUX et E.-M. MEUNIER, « Du religieux en émergence ", dans Sociologie et sociétés, XXV/1, printemps 1993, p. 129.

39 Jean-Paul RoulEAU, "Le catholicisme, vingt-cinq ans après Vatican II ", Sociologie et sociétés XXII/2 (1990) p. 44; R. LEMIEUX et J.P. MONTMINY, op. cit., p. 586. 
donner un sens à la vie, un soutien dans les épreuves, un espoir d'aprèsmort et un ensemble de grands principes moraux; ils comptent que le contenu soit articulé autour de l'« histoire de Jésus". "Toutefois, comme les convictions du parent reposent sur une appropriation sélective de certains éléments de l'héritage religieux, comportant de ce fait l'évacuation d'un certain nombre d'adhésions à l'un ou l'autre aspect de la doctrine, de la morale ou des pratiques proposées par l'Église, la parent s'attend à ce que l'enseignement dispensé à l'école n'ignore pas cette distance 40 ". Dans un autre exposé Milot avertit que si l'on fait référénce à une communauté religieuse, c'est d'une "communauté virtuelle » qu'il s'agit comme étant "l'extension imaginaire du "foyer de sens" propre à chacun des individus, foyer qui peut prendre appui de manière sélective sur des références et des pratiques religieuses de la communauté d'appartenance, et ce à divers degrés et jusqu'à l'appropriation non religieuse de certaines pratiques 41 ". Dans son commentaire du sondage SOM auprès des Montréalais, P.-A. Turcotte privilégie plutôt le diagnostic d'une religion populaire de masse, remarquable par l'insertion périodique dans une tradition, où les compromis existentiels des engagements sont variés et où se manifeste la protestation laïque contre l'enserrement de l'expression religieuse dans une ritualité définie et réformée par les clercs 42 . Enfin, l'enquête dirigée par J. Grand'Maison fait voir que la recherche ne peut se limiter aux questions explicitement religieuses. La crise du croire est d'abord au plan séculier. Mais alors beaucoup d'adultes n'arrivent pas à ressaisir leur vie séculière dans une perspective de foi chrétienne, laquelle est souvent ramenée à une foi infantile de dépendance qui heurte les valeurs d'autonomie et de liberté de conscience. Les jeunes ont devant eux peu de modèles adultes qui présentent une véritable structuration intégrée de la vie séculière, de l'expérience intérieure et de la foi chrétienne ${ }^{43}$.

40 Micheline MILOT, Une religion à transmettre? Le choix des parents. Essai d'analyse culturelle. Québec, Presses de l'Université Laval, 1991, p. 75, 127-128.

41 M. MiLoT, «Pluralisme religieux et univers scolaire montréalais", dans Société, culture et religion à Montréal, p. 145.

42 P.-A. TURCOTTE, «Catholicisme romain ou catholicisme culturel ", dans Société, culture et religion à Montréal, p. 178 ss.

43 Jacques GRAND'MAISON, Le drame spirituel des adolescents. Profils sociaux et religieux, (Cahiers d'études pastorales, 10). Montréal, Fides, 1991, p. 129. 


\section{La mission chrétienne en ville}

Le christianisme, au nom du pluralisme même, est justifié d'occuper sa place et d'offrir sa contribution au service de la ville. Le mouvement chrétien tient sa mission de l'œuvre de Jésus Christ dont le témoignage, les actions et les enseignements consistent essentiellement en l'édification du "Règne de Dieu" : "aux autres villes aussi il me faut annoncer la bonne nouvelle du Règne de Dieu, car c'est pour cela que j'ai été envoyé ", dit Jésus à la foule ( $L c 4,43$ ). La catégorie biblique du Règne désigne l'accomplissement de la création selon l'intention de Dieu. Décrit dans ses effets tangibles, c'est le règne de la réconciliation, de l'amour, de la dignité humaine rétablie, de la justice, de la paix; c'est l'humanité rendue neuve, en voie d'atteindre sa pleine humanité. Ce n'est pas vaine imagination car son avènement est illustré concrètement par la praxis de Jésus, lequel incite les hommes et les femmes à faire de même: le Règne devient un projet à réaliser, impliquant des attitudes et des pratiques. Cette dynamique du temps de l'histoire est promise au Royaume définitif dont une des figures est la "Jérusalem nouvelle" (Ap 21,2), la cité eschatologique. Le Règne achevé embrasse tout et donne la signification voilée de l'histoire de la libération intégrale ${ }^{44}$. Depuis Jésus le temps attendu de la fin est commencé et la cité terrestre restaurée peut en être l'ébauche. La mission doit donc travailler petit à petit à faire aboutir le monde à son authentique mondanité, la ville à sa pleine réalisation d'espace humain, les personnes à leur propre accomplissement. Il ne s'agit pas de refaire une cité chrétienne théocratique mais d'exploiter la mission urbaine d'un mouvement qui fournit sa contribution pour porter la ville à son être et à sa finalité propres.

Le contexte urbain donne toutes ses chances à l'avènement de la personne et à une plus grande personnalisation de ses relations sociales car le milieu ne s'interpose, ni ne contraint. Renvoyé à lui-même et à l'apprentissage d'une vie commune d'interdépendance, le citadin a à concilier deux tendances, l'individualisme et la solidarité. Forcé de faire sa synthèse vitale au sein de la mobilité et de la diversité, il doit se donner une solide personnalité en retournant à son intériorité. La mission chrétienne ne peut que promouvoir l'éthique de la réalisation de soi. Elle interpelle

La Jérusalem nouvelle a souvent été identifée à l'Église des derniers temps, depuis l'époque patristique. Or, il s'agit d'une réalité de plus large déploiement. Le Christ va plutôt chercher l'Église pour l'introduire à titre de fiançée dans le Royaume achevé ( $A p 21,9 s s)$. Royaume et Église ne sont pas identiques. Cf. R. SCHNACKENBURG, Règne et royaume de Dieu. Paris, Orante, 1965, p. 282 ss. 
l'individu à poursuivre une démarche de spiritualité pour qu'il oriente et unifie sa vie en rapport avec un horizon de signification. Ce qui implique une recherche qui conduit à la découverte d'une foi et à sa reconnaissance en option personnelle. Or plusieurs sont enclins à se référer à des croyances en vrac, éparpillés et ballottés au gré des opinions. Les croyances doivent alors être évaluées à la force de personnalisation et à l'engagement qu'elles suscitent pour être de réelles instances de spiritualité. L'enjeu est que la position de chacun puisse atteindre le statut d'une foi, c'est-à-dire d'une option de conscience sur un sens déterminant, que cette option soit humaniste, chrétienne ou d'une autre tradition religieuse.

Une telle interpellation est déjà cuvre d'humanisation, sans apologie confessionnelle. Bien entendu, la mission chrétienne saura, en outre, proposer l'offre de sens articulée autour des faits er gestes de Jésus, de ses paroles sur le sens de Dieu et de l'humain, du projet évangélique donné à expérimenter et dont la substance tient dans le projet du Règne de Dieu. Dès lors, toute présentation de contenus doit préférer au modèle doctrinal le modèle herméneutique comme mise en corrélation, chez le sujet, de l'expérience chrétienne fondamentale avec l'expérience humaine d'aujourd'hui : la foi chrétienne est une expérience à vivre à même les multiples expériences séculières et quotidiennes et peut s'avérer l'axe d'un projet de vie. Les agents d'Église sont disponibles pour accompagner, mais sans penser détenir ni le contrôle, ni le monopole. Il appert que beaucoup de gens vivent une marginalité ecclésiale de nature mystique : ils sont des chercheurs inquiets du Dieu caché et vérifient en silence la crédibilité de leur foi. L'expérience mystique a souvent été dans l'histoire " une fracture dans le corps institué autosuffisant », ... façon de lui dire qu'il n'est pas le tout du corps ecclésial ${ }^{45}$. Les médiations premières sont bien l'expérience du sujet, la question de Dieu et l'accès à l'homme Jésus des récits évangéliques. Ainsi la foi et l'identité chrétiennes ne sont conditionnés ni ne doivent s'exprimer par une dépendance obligée envers l'Église comme institution médiatisante d'encadrement. Il faut encore respecter, voire faciliter, les étapes psychologiques de développement de la foi observables chez des gens qui, dépris d'un conformisme d'appartenance, traversent une phase individualisée d'évaluation critique et de départage pour arriver à la réappropriation d'une synthèse personnelle ${ }^{46}$.

45 Gérard ROLLAND, «Le point aveugle, le corps manquant », Concilium 254 (1994) p. 124.

46 Voir James W. Fowler, Stages of Faith. The Psychology of Human Development and the Quest for Meaning. San Francisco, Harper \& Row, 1981. 
L'individu ne devient humain qu'avec les autres, dans cette totalité qu'est "l'universalité des citoyens". "Le seuil de l'humanité, c'est le seuil de la citoyenneté, et le citoyen n'est citoyen que par la Cité ${ }^{47}$ ". La ville fait le citoyen et le destine à vivre en communauté humaine. Réciproquement, les citoyens sont solidairement responsables de faire leur ville. L'art de vivre chrétien, de soi en connivence avec cette intention de solidarité, doit assumer la ville et contribuer spécifiquement à en sauvegarder la visée humanitaire. Son approche est avant tout positive : la mobilité urbaine libère; la diversité des contacts et activités aide au développement des virtualités humaines; la consommation n'est pas qu'avilissante mais fournit des moyens de bien-être, agrémente, crée des styles et des rituels empreints de gratuité; la production, au delà de l'utile, met au monde des objets qui améliorent la qualité de vie; la technologie fait reculer les conditions de maladie, de misère et de pauvreté. En contrepartie, ces facteurs amènent de nouveaux maux, tels l'asservissement aux objets, la manipulation, la discrimination. La vigilance doit s'exercer en regard de l'égalité des chances au travail, de la justice distributive, de la régulation des intérêts de marché, de la prévention des désordres sociaux. Par ailleurs, les relations fonctionnelles entre professionnels et clients, vendeurs et acheteurs, entreprises et ouvriers, se justifient pour la compétence et l'efficacité des interventions et "ne doivent pas être considérées comme humainement pauvres 48 ». Cependant tout ne peut être mesuré à l'étalon de la rationalité instrumentale de la performance maximale ou de la froide objectivation de la gestion. Il s'agit de savoir si l'on tient compte des personnes, les rejoint et les valorise.

Dans l'univers urbain, qui est autrui? Les relations au prochain se manifestent autant envers celui que l'on atteint à travers sa fonction sociale ou par le canal des institutions - le socius selon Ricœur - qu'envers celui que l'on atteint dans la rencontre interpersonnelle. "Il est illusoire de vouloir transmuter toutes les relations humaines dans le style de la communion. L'amitié et l'amour sont des relations rares qui naissent dans les intervalles de relations plus abstraites, plus anonymes 49 ". La charité peut être cachée dans l'humble service fonctionnel à travers le travail, l'éducation, la profession, le commerce. Chacun sera jugé finalement sur ce qu'il aura fait à des personnes, même sans le savoir ( Mt 25, 31s).

Paul RICCEUR, commentant la philosophie politique d'Aristote, dans Histoire et wérité. $3^{e}$ éd., Paris, Seuil, 1964, p. 263. 
Enfin il y a les relations de groupes spontanés et de groupes formels d'échange ou d'action qui confortent les consciences individuelles et façonnent une conscience collective de solidarité. Beaucoup de chrétiens s'engagent à titre personnel dans les organismes communautaires ou les causes de libération, voire en politique. La pratique chrétienne est d'abord une pratique éthique, relationnelle et sociale, avant d'être religieuse ( $M c$ 7,1-23 ). Et les croyants sont souvent disséminés dans la ville pour ce faire. La présence prévaut sur la visibilité.

Les contemporains auront aussi profit à se réapproprier cette solidarité que représente l'Église portée par la foi des chrétiens, qui les a faits se comprendre comme inscrits dans la suite du mouvement communautaire lancé par Jésus. Ils sont "le peuple qui produira les fruits du Règne de Dieu " (Mt 21,43) : l'Église est la communauté voulue pour expérimenter le nouvel ordre de vie, en être le symbole, et prendre le relais de la mission. Douée de charismes divers dévolus à ses membres, elle s'est organisée et a défini son champ institutionnel, puisqu'un corps stable et durable ne peut se dispenser du statut d'institution ${ }^{50}$. Mais l'Église est avant tout un corps social, une communauté spirituelle habitant un lieu. Or, depuis l'origine, elle prend le nom de la ville qu'elle habite car l'Église locale est l'Église de la ville, la communauté chrétienne de telle ville. Dans la ville d'aujourd'hui, l'Église est une large communauté de référence où les membres réfèrent à une origine commune, une foi, des croyances et des ritualités communes, une mémoire collective, qui sont médiatrices de l'expérience de chacun. Elle n'est pas limitée aux communautés constituées qui entretiennent des interrelations fortes et des interactions explicites. On ne peut déprécier en cela le catholicisme culturel qui représente un fond, une assise stable dans une configuration mouvante. On dira qu'il y faut des rassemblements concrets pour que cela ne soir pas que diffus. La célébration des rites de passage (baptême, mariage, mort et adieu au défunt, etc. ) en est une occasion privilégiée chez la majorité. S'y trouve réuni le clan familial, la famille élargie de la parenté, de proches amis et de voisins sélectionnés. En ville, c'est la seule communauté naturelle qui tienne (alors que la famille nucléaire est souvent éclatée) : c'est dans ce réseau de la maisonnée que s'affirment ordinairement les dispositions les plus ouvertes et se transmettent les significations éprouvées. Les rites inscrivent dans une tradition, reliée à l'héritage familial, qui assure la

50 C'est une anomalie historique que des clercs aient fini par avoir la main haute sur l'institution au point de la monopoliser. L'institution n'est pas l'apanage du clergé. Elle est bien plutôt l'ensemble des moyens que l'Église a reçus et qu'elle continue d'élaborer pour assurer sa cohésion, son champ d'expérience et sa mission. 
continuité de la mémoire chrétienne: ils réaffirment la référence à la pérennité d'une lignée croyante ${ }^{51}$. Du fait que ces rites sont vécus en famille, la référence personnalisée à la tradition chrétienne redit le lien d'une communauté croyante de type domestique.

Au plan des structures, le phénomène urbain oblige à diverses modalités réajustées de regroupement ecclésial. La modalité traditionnelle est la paroisse, communauté stable, accessible au tout-venant, repérable en un endroit précis où l'assemblée publique est possible. Personne ne nichant nulle part à moins de s'éparpiller dans l'indétermination, elle peut être pour plusieurs un base d'ancrage communautaire, mais elle est tenue de rester en cela accueillante aux différences de situations spirituelles et de degrés d'appartenance. Si elle doit faciliter l'exercice de rôles engageant de réelles responsabilités, il serait irréaliste de vouloir imposer à tous l'exigence de l'interaction. La paroisse demeure, pour le catholique standard, un centre de services religieux, fonction qu'elle doit remplir dans l'univers urbain des spécialisations. Cependant, de modèle rural transplanté en ville, la paroisse territoriale ne recouvre plus aujourd'hui l'unité de vie collective et elle provoque un effet de cloisonnement. Elle ne peut répondre à tous les besoins religieux ni le faire avec compétence en tout. Alors relative, elle doit s'ouvrir aux groupes sélectifs et à l'Église de la ville.

Une deuxième modalité est celle des communautés de base, dont le prototype est la maisonnée antique, qui correspond bien au trait de la postmodernité favorisant les groupes de pairs, la convivialité et la participation. Cette formule est retenue pour des regroupements de fraternité évangélique ou porteurs de projets communs en milieux populaires. Mais on encourage surtout les petits groupes volontaires de réflexion ou d'action. Des chrétiens éprouvent le besoin de se recomposer, de vérifier leurs croyances avec d'autres, d'intégrer leur foi et leur projet de vie. Ils peuvent jouer un rôle-clé pour la réélaboration nécessaire à la survie des références chrétiennes dans la culture urbaine. D'autres se consacrent à l'intervention: groupes de soutien pour parents d'adolescents, groupe d'entraide de familles monoparentales, "maisons de quartier" offrant aux pauvres et aux exclus des services comme l'accueil, la relation d'aide, la cuisine collective, l'atelier de couture, l'apprentissage de la réinsertion sociale.

51 Danièle HERVIEU-LÉGER, La religion pour mémoire. Paris, Cerf, 1993, p. 119 et 236. A. Charron, « Famille et communauté chrétienne : leur articulation autour des rites ", Liturgie, foi et culture 28/140 (1994) p. 27-33. 
Une troisième modalité est l'Église de la ville, à l'échelle de la ville comme phénomène global de la vie d'ensemble où se passent les échanges de toutes sortes. Le lieu de la communauté chrétienne en ville, c'est aussi la ville elle-même ${ }^{52}$. L'individu y est mobile : il va à la messe en telle église, célèbre son mariage en tel endroit de culte, trouve conseil spirituel auprès de tel agent, cherche information en tel centre, écoute telle émission religieuse à la radio et à la télévision, participe aux activités de tel cercle. Il bénéficie d'un grand nombre de mouvements, associations, institutions, points de service à caractère religieux. Il peut rejoindre des groupes communautaires et des organismes humanitaires de l'organisation civile. Bref, il œuvre ici et là, exerce sa vie chrétienne et prend son ressourcement en différents endroits d'un même lieu, l'Église urbaine ou régionale. Il réalise son appartenance par plusieurs canaux de communication, par des relations fonctionnelles et par des liens sociaux diversifiés.

Ces modalités structurelles ne s'excluent pas et peuvent donc coexister. J'insisterai sur la dernière, la plus neuve, trop oubliée et mal servie. "L'Église de la ville " est un lieu ecclésial pleinement public à exploiter. Il s'agit du lieu séculier d'émergence de l'Église, hors du système paroissial. L'unité pastorale globale est alors l'Église diocésaine ou encore l'Église d'une ville. Une mission urbaine (ou régionale dans les campagnes réunies ) devrait établir des réseaux de solidarité, des passerelles de communication et de concertation entre les chrétiens dispersés et les mouvements et services souvent isolés. Elle pourrait inventer de nouveaux endroits de rencontres, de débats et de rassemblements publics. Une telle instance assurerait la coordination, la planification et la promotion, dans une logique non de contrôle mais de vision et de responsabilité communautaires. Elle ouvrirait la voie à de nouveaux ministères d'agents laïcs; plusieurs prêtres, du presbyterium de la ville, pourraient sortir du cantonnement paroissial et exercer des ministères spécialisés dans les fonctions religieuses, culturelles ou sociales de l'agglomération urbaine.

L'horizon de l'Église aux dimensions de la ville peut influer sur la réorganisation du dispositif paroissial lui-même. Dans de petites villes, on peut, du point de vue de l'animation, regrouper les paroisses et/ou les équipes paroissiales en une unité collégiale urbaine: cela se légitime dans l'idée ancienne et première de l'expérience paroissiale (une ville, une paroisse, un évêque avec son presbyterium ) qui s'harmonisait à l'unité 371-389. F. HOUTARD et J. REMY, Milieu urbain et communauté chrétienne, p. 289 ss. 
globale de vie collective. Par exemple, dans Farnham, l'équipe paroissiale d'animation recouvrant toute la ville est constituée des équipes des six paroisses et dessert 22000 personnes; dans Joliette et les environs, l'équipe échange son personnel et ses ressources entre les treize paroisses pour desservir les 55000 personnes de l'agglomération. Pour les grandes villes, cette mise en commun interparoissiale ne peut se faire qu'en secteurs, étant entendu que le secteur est davantage qu'un simple forum de parole et de concertation mais une unité locale de partage des ressources et de regroupement des services. Telle paroisse qui se donne des ressources dans un domaine spécialisé assure le service pour l'ensemble du secteur; les gens ou les équipes s'y déplacent. Cette formule paraît préférable à celle de la superparoisse obtenue par voie de concentration consécutive à la fermeture d'autres paroisses mais ne résultant qu'en une structure plus grosse qui reste autosuffisante et enfermée dans un morceau de territoire de juridiction limitée.

L'Église ne peut se contenter d'investir dans le remaniement de ses structures ni dans la construction de ses communautés, car elle n'existe pas pour elle-même. Sa mission est au service du "Règne de Dieu " pour l'épanouissement de la vie sur le terrain de ce microcosme qu'est la ville ${ }^{53}$. Sortir de ses cadres et de ses affaires internes, c'est aussi cela assumer la ville. Elle doit d'abord savoir reconnaître les traces de ce Règne déjà présentes dans la vie familiale, sociale, culturelle et religieuse. Elle doit participer gratuitement à la promotion humaine, sans récupération. Elle a à se déplacer, par ses membres, hors de ses espaces symboliques propres vers les lieux séculiers de vie, de réflexion, de délibération, de service, y partageant les questions et les enjeux de l'existence, les problèmes éthiques, la compassion envers les souffrants de toutes souffrances. Dans cet espace relationnel «autre», il lui faut gagner l'hospitalité, s'accréditer par la pertinence de son action et de son discours. Elle aurait avantage à mieux connaître la culture et le fonctionnement des médias comme véhicule de communication adapté à la mobilité urbaine, ouvert au témoignage instantané mais aussi à l'entrevue et au débat.

Le contexte urbain moderne impose le défi de la révision du langage reçu. Pour la juste saisie des représentations bibliques, le passage du mythologique à l'historique et de l'historique à l'existentiel constitue

Si l'Église est au service du Règne de Dieu, si le Règne de Dieu est un projer de salut sur le monde pour le restaurer dans son authenticité et le préparer à son accomplissement, l'Église est au service du monde pour qu'il s'accomplisse comme monde. La mission de l'Église est d'être au service du monde comme monde. 
toujours une exigence. Puis, dans l'usage des évangiles, les référents bucoliques d'une civilisation agraire - troupeau, brebis, champs, graines, oiseaux et fleurs, désert, barque et pêche - qui ont servi à illustrer des situations universelles dans une expérience culturelle donnée, bien qu'ils conservent un pouvoir d'évocation, gagnent à être transposés pour une interprétation actualisée de réception urbaine. Le film Jésus de Montréal a bien réussi ce jeu: tentation du séducteur au sommet d'une tour d'affaires du centre-ville, choix des disciples en leur milieu de travail banalisé, renversement des tables au temple du star-system, mise à mort en victime des systèmes civil et religieux sur la montagne surplombant la ville et ses ambiguités. Le terme de "pastorale" lui-même a beau évoquer la sollicitude envers les appelés au regroupement chrétien, il véhicule l'image champêtre du pasteur avec ses ouailles, en plus de faire dépendre son registre de signification du personnage du prêtre, chef et guide de communauté. Le terme de "mission" a plus d'à-propos et d'extension : il est centré sur l'envoi au monde, tout en incluant la gouverne pastorale interne; il n'est pas réservé à une classe d'intervenants; il connote une raison d'être, une intention, des orientations et des tâches, à l'instar de ce que les règles organisationnelles modernes commandent de préciser à tout groupe ou organisme.

Plus encore, la compréhension de plusieurs croyances demande à se détacher d'un imaginaire cosmologique de culture ancienne - pensons au récit des origines du monde, aux représentations de l'ascension, de l'assomption, des anges, de la résurrection des corps - pour en retrouver la signification anthropologique. Quant au discours chrétien en général, les impératifs de l'acculturation et de l'activité herméneutique imposent le défi de la traduction, au delà de l'adaptation du vocabulaire, pour un dire nouvellement situé en regard du "croyable disponible" de la culture actuelle, c'est-à-dire réélaboré selon les catégories anthropologiques de la subjectivité, de l'historicité, de l'expérience, de la responsabilité, du procès. Cette entreprise requiert la participation de tous ceux qui éprouvent l'intuition de leur foi au point de jonction des avancées de la modernité, des divers domaines du savoir, des situations inédites et des interrogations lancinantes, ce qui peut obliger jusqu'à la réouverture des contenus. Par exemple, le pouvoir d'intervenir sur la nature sous la conduite d'une raison entrée en débats, puis le dépassement des conditionnements appellent à abandonner la conception physiciste de la nature (intouchable et perçue comme un donné brut directement voulu de Dieu ) et à dépasser les modèles supposés inaliénables de comportements qui lui étaient reliés. S'en trouve écartée la conception d'un Dieu providentialiste ( prédéterminant le jeu des "causes secondes") pour dégager la repré- 
sentation d'un Dieu amour, sujet inconditionné devant des humains libres, perçu dans l'horizon de la gratuité ou mieux de la gracieuseté, offrant sens et possibilité de construire du sens à qui veut accomplir son autonomie en tous les aspects individuels et sociaux de la condition humaine. La tradition chrétienne en voit, certes, le dévoilement dans la figure de Jésus.

Mais à l'ère du pluralisme religieux, cette tradition, devenue religion, prend conscience qu'elle est aussi marquée par la finitude et la relativité en tant qu'elle est historique, à l'instar de toutes les religions historiques qui ont leur configuration particulière de représentations toujours déficientes pour dire ce qu'elles désignent ${ }^{54}$. Dans l'espace urbain pluraliste, la mission passe encore par le dialogue interreligieux pour le partage d'expériences et d'angles de vision diversifiés originant d'une même source qu'est la visée de l'Ultime, car la particularité historique du christianisme ne contient pas de soi les richesses d'autres traditions complémentaires. Ce dialogue spirituel, de fécondation réciproque des différences, achemine vers une vérité partagée, visée, advenante, ouvrant aux virtualités souvent inexplorées de la réalisation humaine. Il appelle à une vérité pratiquée, celle qui se vérifie dans les pratiques de transformation individuelles et sociales. La mission de dimension œcuménique alimente ce mouvement universel de l'humanité qui aspire à ce que les chrétiens entrevoient sous la catégorie de cité eschatologique : la cité promise à son achèvement, symbole du destin humain tout entier.

54 Voir Joseph S. O'LEARY, La vérité chrétienne à l'âge du pluralisme religieux, (Cogitatio fidei 181). Paris, Cerf, 1994. Edward SCHILleBEECKX, «Universalité unique d'une figure religieuse historique nommée Jésus de Nazareth», LavThéolPhil 50/2 (1994) p. 265-281. 